\title{
A mathematical model for the biological treatment of industrial wastewaters in a cascade of four reactors
}

\author{
Rubayyi T Alqahtani ${ }^{\text {a }}$, Mark I Nelson, Annette L Worthy \\ ${ }^{a}$ School of Mathematics and Applied Statistics, University of Wollongong, Wollongong, NSW 2522 Australia \\ Email: rtaa648@uow.edu.au
}

\begin{abstract}
Many industrial processes, particularly in the food industry, produce slurries or wastewaters containing high concentrations of biodegradable organic materials. Before these contaminated wastewaters can be discharged the concentration of these pollutants must be reduced. A method which has been extensively employed to remove biodegradable organic matter is biological treatment. In this process the wastewater (or slurry) is passed through a bioreactor containing biomass which grows through consumption of the pollutants.

The industrial treatment of wastewaters typically employs a reactor cascade. In a reactor cascade of $n$ reactors the effluent stream from the ith reactor in the cascade acts as the feed stream for the $(i+1)$ th reactor, i.e. the next reactor. The efficiency of the reactor cascade may be improved by using a settling unit. The settling unit 'captures' and concentrates the microorganisms in the effluent stream of reactor (i) and recycles it into the influent stream of reactor $(j, j \leq i)$. The benefit of using the settling unit is that it increases the concentration of microorganisms in reactor $\mathrm{j}$, hopefully leading to an improvement in the performance of the cascade. When $\mathrm{i}=\mathrm{j}$ the operation of the settling unit is characterised by a single parameter, the dimensionless recycle parameter, which can take values between zero (no recycle) and one (perfect recycle). When $i<j$ the operation of the settling unit is characterised by two parameters: a concentrating factor $(\mathrm{C})$ and a recycle parameter $(\mathrm{R})$. The maximum value of the concentrating factor that be achieved in a specific settling unit is related to the value of the recycle parameter.

We investigate how recycle affects the performance of a reactor cascade with four reactors. We consider the use of one settling unit. Steady state analysis is used to study and compare the performance for the various reactor configurations.

In the first configuration we consider the scenario in which the effluent stream leaving a settling unit placed around the ith reactor enters the feed stream for the ith reactor. With even one settling unit various configurations can be utilized. For instance, the settling unit can be placed after the first, second, third or fourth reactor. We find that if the settling unit is placed around the final reactor the performance of the cascade is optimised when the settling unit operates with perfect recycle. If the settling unit is placed around one of the other reactors the performance is optimised with a value of the dimensionless recycle parameter less than one.

For the second configuration we consider the scenario in which the settling unit is placed after the fourth reactor and the effluent stream from the settling unit is recycled back into the first reactor. We find that there is a critical value of the residence time. If the residence time is below the critical value then the settling unit improves the performance of the reactor cascade whereas if the residence time is above the critical value the performance of the cascade is reduced compared to that of a cascade without a settling unit.
\end{abstract}

We conclude by noting that the first configuration outperforms the second configuration at high residence time. This is noteworthy as the latter is often used in industry.

Keywords: reactor cascade; Contois growth kinetics; Stirred tank; Water treatment. 


\section{INTRODUCTION}

Many industries discharge polluted wastewater containing a large number of harmful compounds which result in serious damage to many life forms. A solution to this problem is to clean the wastewater prior to discharge. An avenue to do this is to pass the polluted wastewater through a reactor containing biomass which grows through consumption of the pollutant. As result of growth of the biomass, more biomass and products are produced. These products are a mixture of carbon dioxide, methane, water and biological compounds. The simplest way to model the biological treatment of wastewater is to assume that two biochemical processes occur. In the first, the substrate is consumed by the microorganisms to produce more microorganisms and product(s). In the second the microorganism dies. The first process is commonly modelled using the Monod growth rate expression. However, extensive experimental work has shown that the anaerobic and the aerobic degradation of wastewater originating from industrial processes is often better described by the Contois growth rate expression such as $\mathrm{Hu}$ et al. (2002). Thus, in this work the degradation of a biodegradable organic material is represented by the Contois growth rate expression proposed by Contois (1959). Only a few studies have used the Contois model to simulate the operation of a bioreactor such as Nelson et al. (2008) and Nelson and Holder (2009). Nelson et al. (2008) investigated the behavior of a single reactor with recycle. In this study, the best performance of the reactor is obtained in the case with perfect recycle $\left(R_{1}^{*}=1\right)$. Nelson and Holder (2009) studied the behaviour of a reactor cascade of $\mathrm{N}$ reactors without recycle. They found that at high residence times the effluent concentration is given by $S_{n}^{*} \approx \frac{1}{\tau_{t}^{* n}}$, where $\tau_{t}^{*}$ is the total residence time of the cascade.

In this paper we extend earlier work (Nelson et al. (2008); Nelson and Holder (2009)) by studying the behaviour of a cascade with four reactors using the Contois model. We consider two scenarios for the operation of the settling unit in a cascade of four reactors. In the first scenario the settling unit is situated on reactor $i$ and recycles the effluent stream back into reactor i. In the second scenario the settling unit is placed after reactor four and the effluent stream is recycled back into the first reactor. The latter configuration is often used in industry. For the first scenario, the equations in the (i)th reactor are independent of these for the (i+1)th reactor. This makes the analysis of the model easier as the reactor cascade equations 'unzip'. In the second scenario, the equations in the first reactor depend upon those of the final reactor because the substrates and the microorganism in the fourth reactor recycle back to the first reactor. The aim of this paper is to find the reactor configuration that minimises the effluent concentration leaving the final reactor.

\subsection{The dimensional model}

1. Reactor cascade with recycle around each reactor $(i=1,2,3,4)$,

$$
\begin{aligned}
V \frac{d S_{i}}{d t} & =F\left(S_{i-1}-S_{i}\right)-X_{i} \frac{\mu\left(S_{i}, X_{i}\right)}{\alpha}, \\
V \frac{d X_{i}}{d t} & =F\left(\left(1+R_{i-1}\left(1-C_{i-1}\right)\right) X_{i-1}-X_{i}\right)+X_{i} \mu\left(S_{i}, X_{i}\right)-K_{d} X_{i}+F R\left(C_{i}-1\right) X_{i} .
\end{aligned}
$$

2. Reactor cascade with recycle around whole cascade $(n=2,3,4)$,

$$
\begin{aligned}
V \frac{d S_{1}}{d t} & =F\left(S_{0}-S_{1}\right)-V X_{1} \frac{\mu\left(S_{1}, X_{1}\right)}{\alpha}+F R\left(S_{n}-S_{1}\right), \\
V \frac{d X_{1}}{d t} & =F\left(X_{0}-X_{1}\right)+R F\left(C X_{n}-X_{1}\right)+V X_{1} \mu\left(S_{1}, X_{1}\right)-K_{d} V X_{1}, \\
V \frac{d S_{n}}{d t} & =F\left(S_{n-1}-S_{n}\right)-V X_{n} \frac{\mu\left(S_{n}, X_{n}\right)}{\alpha}+F R\left(S_{n-1}-S_{n}\right), \\
V \frac{d X_{n}}{d t} & =F\left(X_{n-1}-X_{n}\right)+V X_{n} \mu\left(S_{n}, X_{n}\right)-K_{d} V X_{n}+F R\left(X_{n-1}-X_{n}\right) .
\end{aligned}
$$

Specific growth rate (Contois models), $\quad \mu\left(S_{i}, X_{i}\right)=\mu_{m}\left(\frac{S_{i}}{K_{s} X_{i}+S_{i}}\right)$,

In the following $\mathrm{i}(i=1,2,3,4)$ denotes the ith reactor in the cascade contain $\mathrm{n}$ reactors with recycle around each reactor. The units that the concentrations of the substrate species, $\mathrm{S}$, and the microorganisms, $\mathrm{X}$, are measured in are denoted by $|S|$ and $|X|$ respectively. The parameters in the model are : $C_{i}$, the recycle concentration factor for the settling unit on reactor i (-); F, the flow rate through the reactor cascade $\left(\mathrm{dm}^{3} \mathrm{day}^{-1}\right) ; K_{d}$, the death 
coefficient $\left(\right.$ day $\left.^{-1}\right) ; K_{s}$, the saturation constant $\left(|X||S|^{-1}\right) ; R_{i}$, the recycle ratio for the settling unit on reactor $\mathrm{i}$ based on volumetric flow rates (-); $S_{i}$, the substrate concentration within the ith reactor of the cascade $(|S|) ; S_{0}$, the concentration of substrate flowing into the first reactor of the cascade $\left(\left|S_{0}\right|\right) ; V$, the volume of the reactor in the cascade $\left(\mathrm{dm}^{3}\right) ; X_{i}$, the cell mass concentration within the ith reactor of the cascade $(|X|) ; X_{0}$, the concentration of the cell mass flowing into the first reactor the cascade $(|X|) ; \mathrm{t}$, the time (day); $\alpha$, the yield factor $\left(|X||S|^{-1}\right)$; $\mu(S, X)$, the specific growth rate model $\left(\mathrm{day}^{-1}\right) ; \mu_{\max }$, the maximum specific growth rate (day $\left.{ }^{-1}\right)$; and $\tau_{t}=\frac{n V}{F}$, the total residence time (day). For a specific wastewater, a given biological community and a particular set of environmental conditions the parameters $K_{s}, K_{d}, \alpha$ and $\mu_{\max }$ are fixed. The parameters that can be varied are $C_{i}$, $R_{i}, S_{0}, X_{0}$ and $\tau_{t}$. In our numerical simulations we use parameter values for the anaerobic digestion of ice-cream wastewater that proposed by Hu et al.(2002). These are: $\alpha=0.2116(\mathrm{gVSS})(\mathrm{g} \mathrm{COD})^{-1}, \mu_{\max }=0.9297\left(\mathrm{day}^{-1}\right)$, $K_{d}=0.0131\left(\mathrm{day}^{-1}\right)$ and $K_{s}=0.4818(\mathrm{COD})(\mathrm{g} \mathrm{VSS})^{-1}$. The maximum value of the concentrating factor $C_{i, \max }$ is given by, $C_{i, \max }=\left(1+\frac{1}{R_{i}}\right)$.

\subsection{The dimensionless model}

The system of differential equations, (1-6) can be written in dimensionless form by introducing dimensionless variables for the substrate concentration $\left[S_{i}^{*}=\frac{S_{i}}{S_{0}}\right]$, the microorganism concentration $\left[X^{*}=\frac{K_{S} X_{i}}{S_{0}}\right]$, and time $\left[t^{*}=\mu_{\max } t\right]$. We obtain,

1. Reactor cascade with recycle around each reactor $(i=1,2,3,4)$,

$$
\begin{aligned}
\frac{d S_{i}^{*}}{d t^{*}} & =\frac{n}{\tau_{t}^{*}}\left(S_{i-1}^{*}-S_{i}^{*}\right)-\frac{S_{i}^{*} X_{i}^{*}}{\alpha^{*}\left(S_{i}^{*}+X_{i}^{*}\right)}, \\
\frac{d X_{i}^{*}}{d t^{*}} & =\frac{n\left(\left(1-R_{i-1}^{*}\right) X_{i-1}^{*}-X_{i}^{*}\right)}{\tau_{t}^{*}}+\frac{S_{i}^{*} X_{i}^{*}}{\left(S_{i}^{*}+X_{i}^{*}\right)}+\frac{n R_{i}^{*} X *_{i}}{\tau_{t}^{*}}-K_{d}^{*} X_{i}^{*} .
\end{aligned}
$$

2. Reactor cascade with recycle around whole cascade $(n=2,3,4)$,

$$
\begin{aligned}
\frac{d S_{1}^{*}}{d t^{*}} & =\frac{n}{\tau_{t}^{*}}\left(1-S_{1}^{*}\right)-\frac{S_{1}^{*} X_{1}^{*}}{\alpha^{*}\left(S_{1}^{*}+X_{1}^{*}\right)}+\frac{n R}{\tau_{t}^{*}}\left(S_{2}^{*}-S_{1}^{*}\right), \\
\frac{d X_{1}^{*}}{d t^{*}} & =-\frac{n X_{1}^{*}}{\tau_{t}^{*}}+\frac{S_{1}^{*} X_{1}^{*}}{\left(S_{1}^{*}+X_{1}^{*}\right)}+\frac{n R}{\tau_{t}^{*}}\left(C X_{n}^{*}-X_{1}^{*}\right)-K_{d}^{*} X_{1}^{*}, \\
\frac{d S_{n}^{*}}{d t^{*}} & =\frac{n(1+R)}{\tau_{t}^{*}}\left(S_{1}^{*}-S_{n}^{*}\right)-\frac{S_{n}^{*} X_{n}^{*}}{\alpha^{*}\left(S_{n}^{*}+X_{n}^{*}\right)}, \\
\frac{d X_{n}^{*}}{d t^{*}} & =\frac{n(1+R)\left(X_{1}^{*}-X_{n}^{*}\right)}{\tau_{t}^{*}}+\frac{S_{n}^{*} X_{n}^{*}}{\left(S_{n}^{*}+X_{n}^{*}\right)}-K_{d}^{*} X_{n}^{*} .
\end{aligned}
$$

In equations (7-12), $K_{d}^{*}$ is the dimensionless death rate $\left[K_{d}^{*}=\frac{K_{d}}{\mu_{\max }}\right], R_{i}^{*}$ is the effective recycle parameter around the ith reactor of the cascade $\left[R_{i}^{*}=R_{i}\left(C_{i}-1\right)\right], X_{0}^{*}$ is the dimensionless microorganism concentration in the feed $\left[X_{0}^{*}=\frac{X_{0}}{\alpha K_{s}}\right], \alpha^{*}$ is the dimensionless yield coefficient $\left[\alpha^{*}=K_{s} \alpha\right]$ and $\tau_{t}^{*}$ is dimensionless total residence time $\left[\tau_{t}^{*}=\frac{n V \mu_{\max }}{F}\right]$. All parameters in the model are strictly non-negative. This model is investigated with the assumptions that: $\left[X_{0}^{*}=X_{0}=0\right],\left[S_{0}>0\right]$ and $\left[K_{d}^{*}>0\right]$. When scenario 1 is used, the operation of the settling unit is characterised by a single parameter $R_{i}^{*}$. The maximum value of $R_{i}^{*}$ is given by $R_{i, \max }^{*}=1$. The cases $\left(R_{i}^{*}=0\right),\left(0<R_{i}^{*}<1\right)$ and $\left(R_{i}^{*}=1\right)$ represent no recycle around reactor $\mathrm{i}$, imperfect recycle around reactor $\mathrm{i}$ and perfect recycle around reactor i. When scenario 2 is used, the operation of the settling unit is characterised by two parameters: a concentrating factor $(\mathrm{C})$ and a recycle parameter $(\mathrm{R})$. The maximum value of the concentrating factor that be achieved in a specific settling unit is related to the value of the recycle parameter.

A feature of our dimensionless scheme is that there is a one to one relationship between our dimensionless variable and their dimensional counterparts.

\section{RESUlts}

The steady-state solutions of (7-8) and their stability are readily found by a series of straight-forward manipulations. The physically meaningful steady-state solution of (9-12) and their stability has to be determined numerically. The solution was found using the maple solve command. Steady state diagrams showing the variation of the effluent concentration $\left(S_{e}^{*}\right)$ as a function of the total residence $\left(\tau_{t}^{*}\right)$ time are plotted. It should be noted that only the stable physical meaningful solutions are presented. 


\subsection{Reactor Cascade With Recycle Around Each Reactor (7-8)}

Figure 1 shows the effluent concentration $\left(S_{e}^{*}=S_{4}^{*}\right)$ in a cascade of four reactors for the cases when there is either perfect recycle around each reactor $\left(R_{i}^{*}=1\right)$ or no recycle around each reactor $\left(R_{i}^{*}=0\right)$, for a single reactor with perfect recycle $\left(R_{1}^{*}=1\right)(i=1,2,3,4)$. For a cascade of four reactors, the performance of the flow reactor with perfect recycle is superior to that of the flow reactor with no recycle but the difference in performance reduces as the total residence time increases. The effluent concentration leaving the reactor cascade with perfect recycle around each reactor decreases more rapidly than the effluent concentration leaving the single reactor with perfect recycle. Comparing the single reactor with perfect recycle and the reactor cascade with no recycle, there are two distinct regions. In the first region, $\left(0 \leq \tau_{t}^{*}<4.1\right)$, the effluent concentration leaving the single reactor is lower than that leaving the cascade reactor of four reactors with no recycle. In the first region the performance of a single reactor with perfect recycle is superior to the reactor cascade of four reactors with no recycle. In the second region, $\left(\tau_{t}^{*}>4.1\right)$, the effluent concentration leaving the four reactors cascade is lower than that leaving the single reactor.

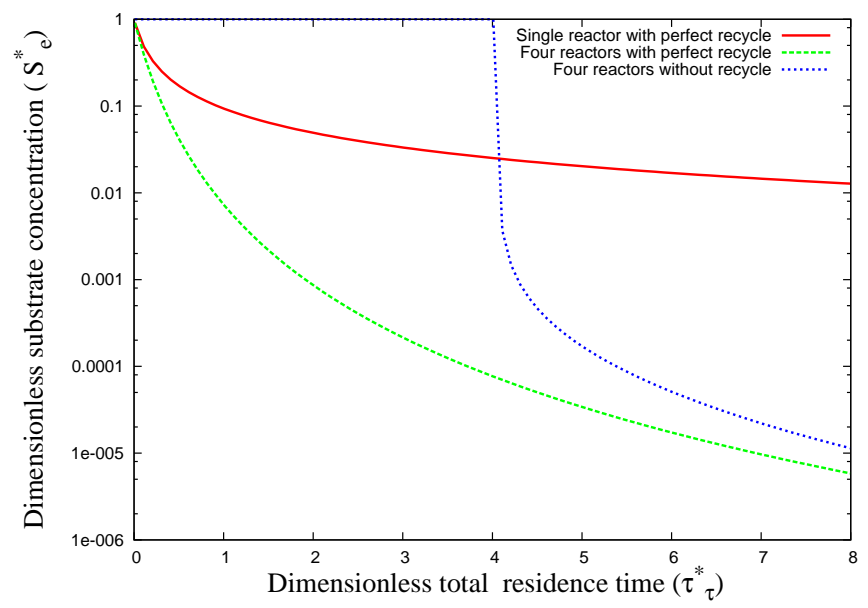

Figure 1. Effluent concentration in a cascade of four reactors and a single reactor as a function of $\tau_{t}^{*}$.

Recycle Around the fourth Reactor. It can be shown that when there is a setting unit with recycle around the fourth reactor the effluent concentration is minimized with perfect recycle in the setting unit $\left(R_{4}^{*}=1\right)$.

Recycle Around the first Reactor. We consider the performance of a cascade contain four reactors when the recycle parameter in the first reactor $\left(R_{1}^{*}\right)$ is varied for a fixed value of the recycle parameter in the second, third, and fourth reactor. Figure 2 shows the performance of the cascade as the value of $R_{1}^{*}$ is varied.

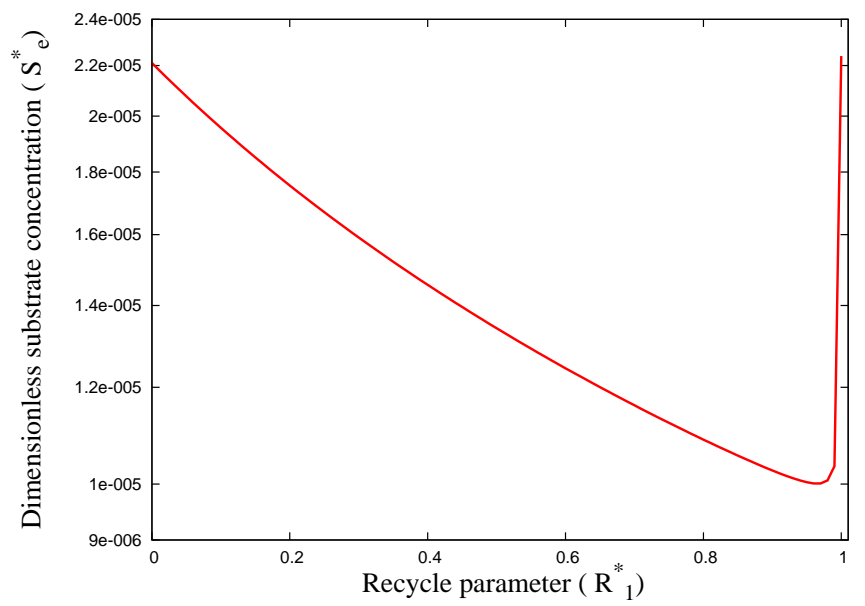

Figure 2. Effluent concentrations as a function of $R_{1}^{*}$. Parameter value: $R_{i}^{*}=0, \mathrm{i}=2,3,4 . \tau_{t}^{*}=7$. 
In this cases there is an optimal value of $R_{1}^{*}, R_{1, \min }^{*}$, at which the effluent concentration is minimized. It is observed that, in this figure $2, R_{1, \min }^{*}<1$. A second feature of this diagram is that there is a critical value of $R_{1}^{*}, R_{1, \max }^{*}$. When $0<R_{1}^{*}<R_{1, \max }^{*}$, recycle has a positive effect upon the effluent concentration whereas if $R_{1, \max }^{*}<R_{1}^{*}<1$ then recycle has a negative effect upon the effluent concentration. Figure 2 shows that the performance of a cascade with perfect recycle around the first reactor $\left(R_{1}^{*}=1\right)$ is slightly inferior to that of a cascade with no recycle around the first reactor $\left(R_{1}^{*}=0\right)$. The critical value $\left(R_{1, \max }^{*}\right)$ only exists for particular value of the dimensionless effective recycle parameter $\left(R_{i}^{*}, i=2,3,4\right)$. For example, when $\tau_{t}^{*}=7$, the critical point $\left(R_{1, \max }^{*}\right)$ only exists when the value of the dimensionless effective recycle parameter $\left(R_{i}^{*}, i=2,3,4\right)$ is sufficiently small. Figure 2 shows a surprising result namely that the optimal performance of a cascade with recycle around each reactor is not obtained with perfect recycle around each reactor $\left(R_{i}^{*}=1\right)$. It is given instead by $R_{4}^{*}=1, R_{i}^{*}=R_{i, \min }^{*}, i=1,2,3$, where the values $R_{i}^{*}, i=1,2,3$ must be determined for each value of $\tau_{t}^{*}$. This outcome is a result of two competing processes. As the value for $R_{i-1}^{*}$ increases, the microorganisms concentration $\left(X_{i-1}^{*}\right)$ entering the ith reactor, the term $n\left(1-R_{i-1}^{*}\right) X_{i-1}^{*}$ in (8), decreases. At the same time, the substrate concentration $\left(S_{i-1}^{*}\right)$ entering the ith reactor also decreases. These processes have opposing effects upon the effluent concentration leaving the ith reactor. Over most values of $R_{i-1}^{*}$ the later effect dominates and the effluent concentration decreases in the ith reactor. However, when the value of $R_{i-1}^{*}$ the former effect is close to 1 dominates and the effluent concentration increases. Thus there is a competition in the ith reactor between the benefits of reducing the dimensionless substrate concentration entering this reactor and disadvantages of reducing the dimensionless microorganism concentrations entering this reactor.

Reactor Configurations. Six reactor configurations are considered (scenario 1). These are:

(a) Configuration : No recycling around each reactor.

(b) Configuration : Optimized recycle around the first reactor and no recycling around the ith reactor.

(c) Configuration : Optimized recycle around the second reactor and no recycling around the ith reactor.

(d) Configuration : Optimized recycle around the third reactor and no recycling around the ith reactor.

(e) Configuration : Optimized recycle around the fourth reactor and no recycling around the ith reactor.

(f) Configuration: The optimized cascade.

The comparison of these reactor configurations is demonstrated at figure 3 . The solution curves in figure 3 splits into two components. The component with lower effluent concentration contains the optimized cascade (f) and the cascade with optimized recycle around the first reactor (b). The performance of configuration (f) is slightly superior to the performance of configuration (b).

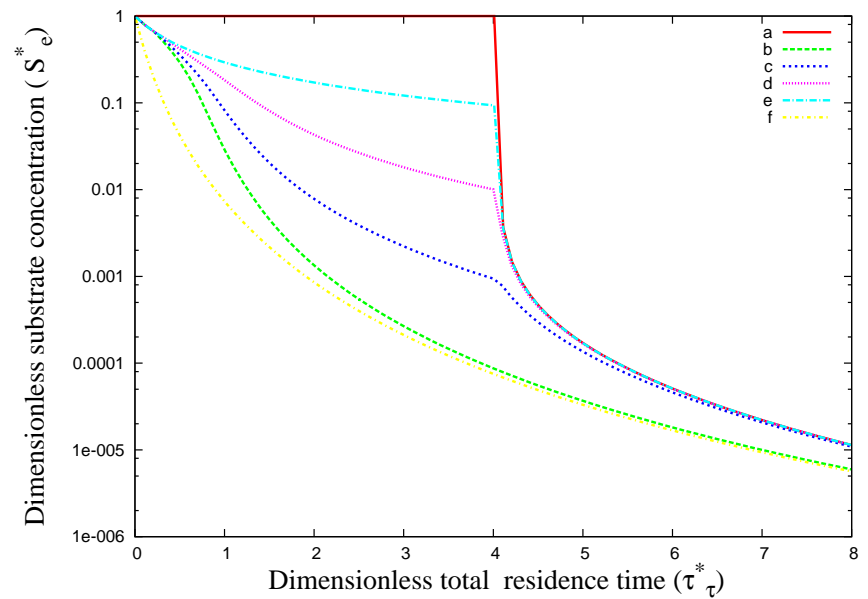

Figure 3. Effluent concentration in a cascade of four reactors as a function of total residence time (scenario 1) where (a) $R_{i}^{*}=0$, (b) $R_{1}^{*}=R_{1, \text { opt }}^{*}, R_{i}^{*}=0$, (c) $R_{2}^{*}=R_{2, \text { opt }}^{*}, R_{i}^{*}=0$ (d) $R_{3}^{*}=R_{3, \text { opt }}^{*}, R_{i}^{*}=0$ and (e) $R_{4}^{*}=1, R_{i}^{*}=0$, (f) $R_{i}^{*}=R_{i, \text { opt }}^{*}, R_{4}^{*}=1$. 
For large total residence time $\left(\tau_{t}^{*} \gg \frac{4}{1-K_{d}^{*}}\right)$ the performance of configuration (b) converges to the performance of configuration (f). Thus, there may be little gain from using the two additional settling units required for the optimized cascade. The component with higher effluent concentration contains the configurations where there are one settling unit (c, d and e) or no settling units (a). For small total residence time $\left(\tau_{t}^{*}<\frac{4}{1-K_{d}^{*}}\right)$, the configurations where there are one setting unit, the performance of the reactor cascade with the optimized recycle around the ith reactor improves as the value for i decreases. In this region, the cascade using a single setting unit around any reactor is superior to the cascade with no recycle. For large total residence time $\left(\tau_{t}^{*} \gg \frac{4}{1-K_{d}^{*}}\right)$, the performance of the cascade with optimized recycle around either the second, the third reactor or fourth reactor (c, $d$ and e) is superior to the performance of the cascade with no recycle (a). However, the performance of configuration (a) converges to that of the performance configurations (c, $d$ and e) in the limit of infinite total residence time.

\subsection{Reactor Cascade With Recycle Around the whole Cascades (9- 12)}

Figure 4 shows the effluent concentration as a function of the total residence time when there is recycle around the whole cascade with $R=0,0.5$ and 1 , respectively (scenario 2 ).

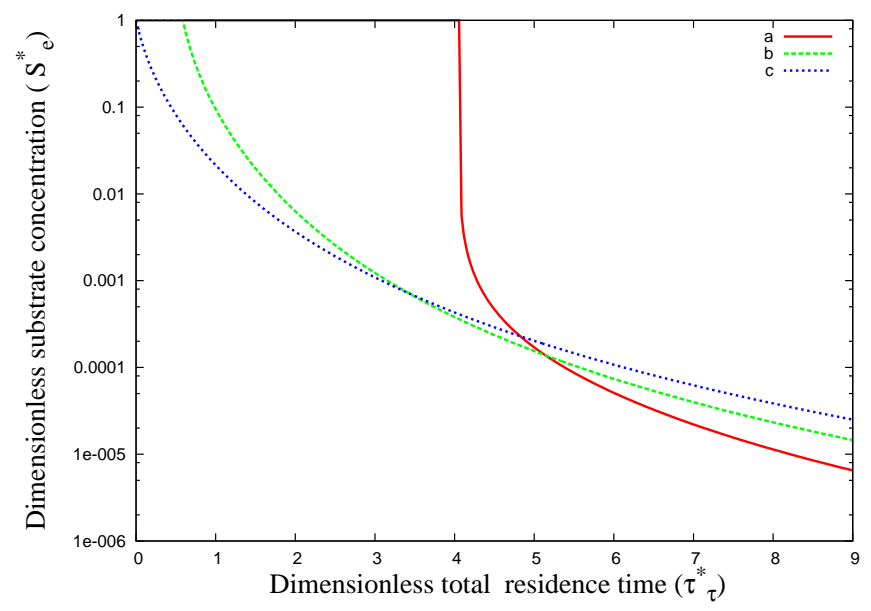

Figure 4. Effluent concentration in a cascade of four reactors with recycles around whole a cascade (scenario 2). The value of parameters: $R=$ (a) 0 , (b) 0.5 and (c) $1, \mathrm{C}=2$.

It can be seen that for each value of $\mathrm{R}(R>0)$ there are three distinct regions. Region 1 corresponds to the case when the washout occurs through the reactor cascade. As $R$ increases from 0 to 1 , the value of the washout point decreases. Region 2 is where the performance of the cascade with recycle is superior to the performance of the cascade without recycle. In this region the performance of the reactor improves as the recycle parameter $(R)$ increases. This region finishes when the effluent concentration curve without recycle $(R=0)$ intersects the effluent concentration curve with recycle. This intersection point marks the point at which the effect of recycle (R) upon the reactor performance changes from positive to negative. Region 3 is where the performance of the cascade with recycle is inferior to than the performance of the reactor cascade without recycle. This is where recycle has negative effect upon the effluent concentration. For a fixed value of total residence time, an increase in the recycle parameter $(\mathrm{R})$ causes an increase in the effluent concentration.

\subsection{Comparisons between the two reactor configurations}

Figure 5 shows that the effluent concentration leaving either a cascade with perfect recycle around the ith reactor (a,b,c and d), $(i=1,2,3,4)$, (scenario 1 ) or a reactor cascade with recycle around the whole cascade (e), (scenario 2). In this figure the choice of the parameter values $(R=1, C=2)$ gives the perfect recycle around the $i$ th reactor. It can be seen that there are three distinct regions. In the first region, the performance of configuration (e) is superior to the performance of configurations (a,b,c and d). In the second region, the performance of configuration (e) is inferior to the performance of configurations ( $a$ and b) but it is superior to the performance of configurations (b and c). In the third region, the performance of configuration (e) is inferior to the performance of configuration (a,b,c and d). Consequently, the choice of reactor configuration depends upon the desired effluent 


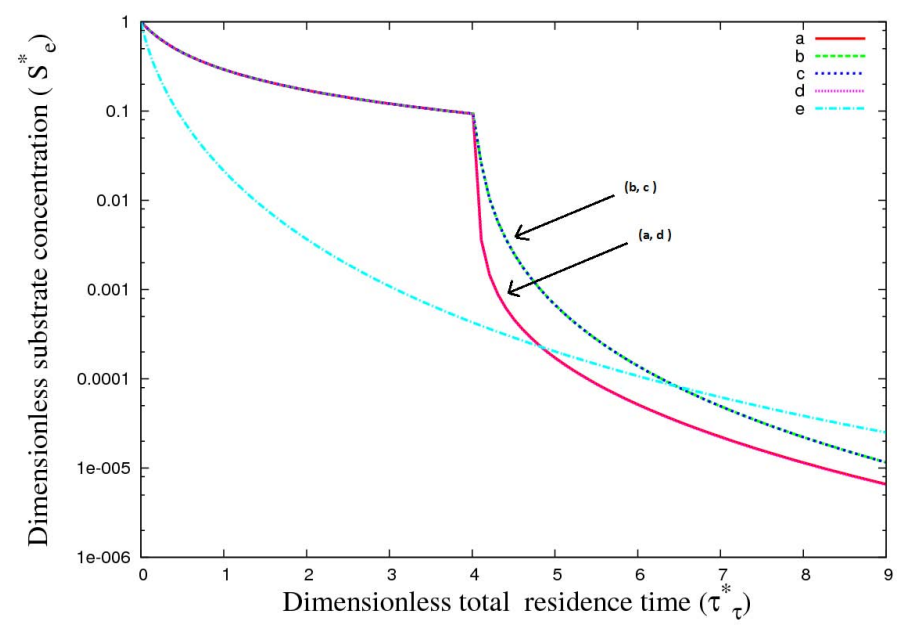

Figure 5. Effluent concentration in a cascade of four reactor for scenario 1 (a) $R_{1}^{*}=1, R_{i}^{*}=0$, (b) $R_{2}^{*}=1$, $R_{i}^{*}=0,(\mathrm{c}) R_{3}^{*}=1, R_{i}^{*}=0,(\mathrm{~d}) R_{4}^{*}=1, R_{i}^{*}=0$, and for scenario 2 (e) $R=1, \mathrm{C}=2$.

concentration. In the first region, scenario 2 should be chosen. In the second region, scenario 1 with perfect recycle around the first or the fourth reactor should be chosen. In the third region, the scenario 1 should be chosen.

\section{CONCLUSION}

In this paper we investigated how the use of settling units effects the behavior of a cascade of four reactors. Two recycle scenarios are considered. When four setting units are deployed in the first scenario, a surprising result is found that the optimized performance of the reactor cascade occurs with perfect recycle around the fourth reactor and imperfect recycle around the ith reactors $(i=1,2,3)$ (Figure 3 ). When only one setting units is employed we found that the performance of the reactor cascade with optimized recycle around the ith reactor is inferior to the performance of that with optimized recycle around the $j$ th reactor at lower total residence times $(j<i)$ (Figure 3 ). For the second scenario (figure 4), there is a critical value of the residence time. If the residence time is below the critical value then the settling unit improves the performance of the reactor cascade whereas if the residence time above the critical values the performance of the cascade is reduced compared to that of a cascade without a settling unit. In figure 5, we compared the the performance of the cascade in the second scenario (turquoise curve (e)) with the performance of the cascade in the first scenario. We found that at low total residence time the performance of the former is superior (scenario 2). At high total residence time, it is better to have recycle around each reactor (scenario 1).

\section{ACKNOWLEDGEMENT}

The first author would like to thank the Saudi government for through the awarding of a PhD Scholarship. He also would like to thank Dr.Nelson and Dr.Worthy for support through his journey.

\section{REFERENCES}

Contois, D. (1959). Kinetics of bacterial growth: Relationship between population density and specific growth rate of continuous cultures. Journal of General Microbiology 21, 40-50.

Hu, C., K. Thayanithy, and C. Forster (2002). A kinetic study of the anaerobic digestion of ice-cream wastewater. Process Biochemistry 37, 965-971.

Nelson, M., E. Balakrishnan, H. Sidhu, and X. Chen (2008). A fundamental analysis of continuous flow bioreactor models and membrane reactor models to process industrial wastewaters. Chemical Engineering Journal 140, $521-528$.

Nelson, M. and A. Holder (2009). A fundamental analysis of continuous flow bioreactor models governed by contois kinetics. ii. reactor cascades. Chemical Engineering Journal 149, 406-416. 